\title{
Sialidosis type 2
}

INSERM

\section{Source}

INSERM. (1999). Orphanet: an online rare disease and orphan drug data base. Sialidosis type 2. ORPHA:87876

Sialidosis type 2 (ST -2) is a rare lysosomal storage disease, and the severe, early onset form of sialidosis (see this term) characterized by a progressively severe mucopolysaccharidosis-like phenotype (coarse facies, dysostosis multiplex, hepatosplenomegaly), macular cherry-red spots as well as psychomotor and developmental delay. ST-2 displays a broad spectrum of clinical severity with antenatal/congenital, infantile and juvenile presentations. 